\title{
The Long-Term Outcomes of Induction Chemoradiotherapy Followed by Surgery for Locally Advanced Non-Small Cell Lung Cancer
}

\author{
Hidetaka Uramoto ${ }^{a}$ Hirohiko Akiyama $^{a}$ Yuki Nakajima ${ }^{a}$ \\ Hiroyasu Kinoshita $^{a}$ Takuya Inoue $^{a}$ Futoshi Kurimoto $^{b}$ \\ Yu Nishimura ${ }^{c}$ Yoshihiro Saito $^{d}$ Hiroshi Sakai $^{b}$ Kunihiko Kobayashi ${ }^{e}$ \\ Divisions of ${ }^{a}$ Thoracic Surgery and ${ }^{b}$ Thoracic Oncology, and Departments of ${ }^{c}$ Pathology \\ and ${ }^{\mathrm{d}}$ Radiation Oncology, Saitama Cancer Center, Saitama, and ${ }^{\mathrm{e}}$ Department of \\ Respiratory Medicine, Saitama International Medical Center, Hidaka, Japan
}

\section{Key Words}

Non-small cell lung cancer · Locally advanced non-small cell lung cancer · Induction chemotherapy · Surgical resection · Prognosis

\begin{abstract}
Background: Although the concept of induction therapy followed by surgical resection for locally advanced non-small cell lung cancer (LA-NSCLC) has found general acceptance, the appropriate indications and the strategy for this treatment are still controversial. Methods: From 2000 through 2008, 36 patients received concurrent chemoradiotherapy followed by surgery. We retrospectively reviewed these cases, analyzed the outcomes and examined the prognosis. Results: The median radiation dose given was $60 \mathrm{~Gy}$. Chemotherapy included a platinum agent in all cases; cisplatin-based chemotherapy was administered to 9 cases, and a carboplatin-based chemotherapy regimen was administered to 27 . A complete resection was performed in $94 \%$ of the patients. Seventeen (47.2\%) patients exhibited a complete pathological response, and downstaging was induced in 26 (72\%) cases. The morbidity and 30-day mortality rates were 11.1 and $0 \%$, respectively. The 5 -year overall survival rate in the patients with complete resection $(n=33)$ was $83.3 \%$. Conclusions: Induction chemoradiotherapy followed by surgery for LA-NSCLC provided a favorable prognosis for selected patients. A complete pathological response was found in about half of cases. This strategy is feasible and was associated with low morbidity and high resectability rates, suggesting that it contributed to improving the treatment results.


Uramoto et al.: The Long-Term Outcomes of Induction Chemoradiotherapy Followed by Surgery for Locally Advanced Non-Small Cell Lung Cancer

\section{Introduction}

Lung cancer is the leading cause of cancer-related death worldwide, and approximately one third of patients with lung cancer are diagnosed to have locally advanced disease [1]. Locally advanced non-small cell lung cancer (LA-NSCLC) has a significantly worse prognosis in comparison to less advanced disease [2]. However, appropriate indications pertaining to surgical treatment in this subgroup of patients are still lacking and are controversial $[3,4]$. The theoretical advantages of preoperative induction therapy lie in a potential increase in the resectability rate, better compliance and a lower rate of distant relapse owing to eradication of micrometastases, both of which may contribute to achieving long-term survival for these patients. Although the concept of induction therapy followed by surgical resection has found general acceptance, it is still controversial in clinical practice from the perspectives of the timing of surgery and the precise procedure [5-7]. Therefore, information about the long-term outcomes and the most appropriate strategy for this subgroup is urgently needed. The aim of this study was to retrospectively identify the outcomes of induction chemoradiotherapy followed by surgery in patients with LA-NSCLC.

\section{Patients and Methods}

\section{Patients}

All patients gave their written informed consent for treatment, with the observational research and privacy policy fully explained. The pretreatment evaluation included a medical history, physical examination, complete blood cell count and serum chemistry data, including serum electrolyte, liver enzyme, bilirubin, creatinine and coagulation values. Patients were eligible for this study if they had histologically or cytologically documented stage III LA-NSCLC that was deemed resectable. Our eligibility requirements included being $<75$ years old, having an Eastern Cooperative Oncology Group performance status of 0-1, no previous chemotherapy or radiotherapy, and adequate pretreatment hematological function, renal function (a normal serum creatinine concentration), hepatic function and pulmonary function. We calculated the predicted residual pulmonary function using the following formula: predicted residual pulmonary function = pulmonary function $\times(1-$ resected pulmonary segments/number of pulmonary segments on the actual state).

Surgery was indicated for patients with a forced expiratory volume in $1 \mathrm{~s}$ of $>600 \mathrm{ml} / \mathrm{m}^{2}$ [8]. Patients were excluded if they had contralateral hilar lymph node metastasis, a serious preexisting disease or a radiation field that exceeded half of one lung. Tumor staging was performed based on chest radiography, a computed tomography (CT) scan of the chest and upper abdomen and a bronchoscopy. Patients underwent a preoperative cardiovascular risk assessment, including ECG and ultrasound cardiography. The patients' records, including their clinical data, preoperative examination results, histopathological findings and the TNM stages were reviewed.

The preoperative assessments included chest roentgenography and CT of the chest, upper abdomen and the brain. Positron emission tomography (PET) scans were used in the assessment of the clinical staging. Magnetic resonance imaging (MRI) of the brain was routinely employed. Mediastinoscopy or endobronchial ultrasound-guided transbronchial needle aspiration was not routinely performed for the evaluation of N2 disease. Most of the cases were suspected to have invasive and extranodal expansion based on the CT and PET findings. 
Uramoto et al:: The Long-Term Outcomes of Induction Chemoradiotherapy Followed by Surgery for Locally Advanced Non-Small Cell Lung Cancer

\section{Treatment Plan}

All patients received preoperative therapy including chemotherapy and concurrent definite radiotherapy. Patients were given docetaxel (DTX) at $30 \mathrm{mg} / \mathrm{m}^{2}$ and carboplatin (CBDCA) at an area under the curve of 3 every 2 weeks for 6 courses [9], or they received cisplatin (CDDP) at $80 \mathrm{mg} / \mathrm{m}^{2}$ and DTX at $60 \mathrm{mg} / \mathrm{m}^{2}$ on day 1 [1], or CDDP at $80 \mathrm{mg} / \mathrm{m}^{2}$ on day 1 and etoposide (VP16) at $100 \mathrm{mg} / \mathrm{m}^{2}$ on days 1-3 [10] of a 21-day treatment cycle for 3 planned cycles.

Radiation treatment planning for all patients was based on CT scans. Radiation therapy was administered with 2.0-Gy daily fractions by a linear accelerator generating 10-MV photons. The initial radiotherapy target volume was the primary tumor, and ipsilateral hilar and mediastinal lymph nodes by anteroposterior parallel-opposed fields with a 1- to $1.5-\mathrm{cm}$ margin around the primary tumor and involved nodes up to $60 \mathrm{~Gy}$, targeted in 6 weeks without interruption. A shrinking field technique was used. Mediastinal lymph nodes were irradiated at up to $40 \mathrm{~Gy}$ for prophylaxis with tissue homogeneity corrections. These doses were calculated at the central axis without the use of lung correction. The paraesophageal nodes were included if the lesion was in the lower lobe. The contralateral hilum was excluded [11].

Routine re-evaluation was carried out according to the New Guidelines for Evaluation of the Treatment Response of Solid Tumors [12]. In principle, the period between the end of the radiation treatment and surgery was at least 8 weeks. Before surgery, a second risk analysis was performed, and the decision regarding surgical intervention was made jointly by a committee including the attending radiation oncologist, thoracic surgeon, medical oncologist and pulmonologist. For example, a case with radiation pneumonitis was excluded. Following re-evaluation, surgery was attempted for patients in whom R0 resection was deemed possible.

All surgeries were performed via open thoracotomy, and systematic mediastinal lymph node dissection (en bloc removal of the mediastinal fatty tissue containing the lymphatics) was performed as well. Extended resection was defined as a procedure that included combined resection of the surrounding structures and a complex lobectomy with reconstruction of the bronchus [13]. A resection was considered to be complete if, after review of the surgery and pathology reports, both surgical margins and the highest mediastinal lymph node were found to be free of tumor cells. The bronchial stump was, in principle, covered with pericardial fat tissue or an intercostal muscle pedicle. The pathological effect of induction therapy was classified according to the General Rule for Clinical and Pathological Record of Lung Cancer, sixth edition [14] as a pathologically complete response (complete cancer cell death; Ef3), a major response (fewer than one third of cancer cells were viable; Ef2) or a minor response (more than one third of cancer cells were viable; Ef1). The patients with complete resection did not receive any additional treatment.

All resected specimens were subjected to a pathological examination according to the 7th edition of the TNM classification as described by the IASLC. Follow-up information was obtained from all the patients through office visits or by telephone interviews. The patients were basically evaluated every 3 months by a physical examination, chest roentgenography, an analysis of the blood chemistry variables and measurements of the tumor marker levels. As a general rule, chest and abdominal CT scans, brain MRI and a bone scintiscan were obtained every 6 months for the first 2 years after surgery and annually thereafter for 5 years or more. Additional examinations were performed if any symptoms or signs of recurrence were detected. Survival was calculated from the date of surgery until death or the date of the last follow-up. 
Uramoto et al.: The Long-Term Outcomes of Induction Chemoradiotherapy Followed by Surgery for Locally Advanced Non-Small Cell Lung Cancer

\section{Statistical Analysis}

The Kaplan-Meier method was used to estimate the probability of survival, and survival differences were analyzed using the log-rank test. Differences were considered to be statistically significant for $\mathrm{p}$ values $<0.05$. The data were analyzed using the StatView software package (Abacus Concepts, Inc., Berkeley, Calif., USA).

\section{Results}

\section{Patient Characteristics}

From 2000 through 2008, 1,121 consecutive patients with lung cancer underwent pulmonary resection at our institution. Of those, 44 LA-NSCLC patients who underwent induction chemoradiotherapy followed by surgery were included in this study. A salvage operation was performed for 8 patients. Therefore, a total of 36 patients $(3.2 \%)$ were evaluable, and their characteristics are shown in table 1 . The patient sample consisted of 32 males and 4 females, with a mean age of 57.6 years (range, 29-74 years). The tumors included 19 adenocarcinomas, 15 squamous cell carcinomas (SQ), 1 adenosquamous carcinoma and 1 large-cell neuroendocrine carcinoma. Eight patients were in stage IIB, 16 in stage IIIA and 12 in stage IIIB.

\section{Clinical Response and Survival}

Twenty-seven patients received CBDCA plus DTX, 8 received CDDP plus DTX and 1 patient received CDDP plus VP16. The cumulative median radiation dose to the primary tumor was 60 Gy (range, 50-60 Gy). The median number of courses of induction chemotherapy was 3. The clinical response to the induction therapy was complete response in 1 patient, partial response in 32 and stable disease in 3 patients.

\section{Surgery and Pathological Response}

The median time from the end of the induction therapy until surgery was 94 days (range, 46-199 days). The operation was performed during a mean of 323 min (range, 190$530 \mathrm{~min}$ ), and the mean blood loss was $407 \mathrm{ml}$ (range, 107-1,098 ml). Only 1 case needed a blood transfusion. The surgical procedures included a lobectomy in 31 patients, bilobectomy in 2 patients, partial resection in 1 patient and a right-sided pneumonectomy in 2 patients. Eight cases and 1 case underwent a lung resection combined with a chest wall resection and azygos vein resection, respectively. Thirty-four patients had a complete tumor resection with microscopically negative margins. The reasons for the incomplete resections $(n=2)$ were as follows: residual cancer cells in the parietal pleura in 1 case and in the mediastinal lymph node in 1 case. Thus, a complete resection was performed in $94 \%$ of the patients.

\section{Pathological Findings}

With regard to the pathological effect of induction therapy, there were $17(47.2 \%)$ patients who exhibited an Ef3 response, 12 who exhibited an Ef2 response and 7 who exhibited an Ef1 response. One case with complete response and 16 cases with partial response $50 \%$ of the 32 partial response cases) also showed an Ef3 response based on the imaging evaluations (table 2). With regard to the stage, downstaging was induced in $26(72 \%)$ patients overall. Downstaging was obtained in $50 \%$ of the IIB patients, in $88 \%$ of the IIIA and in $67 \%$ of the IIIB patients (table 3 ). 
Uramoto et al:: The Long-Term Outcomes of Induction Chemoradiotherapy Followed by Surgery for Locally Advanced Non-Small Cell Lung Cancer

\section{Surgical Morbidity and Mortality}

The morbidity and 30-day mortality rates were 11.1 and $0 \%$, respectively. The morbidity included atelectasis, chylothorax, prolonged air leakage and empyema in 1 case each (table 4). One patient suddenly died from hemoptysis after right upper sleeve lobectomy 34 days after the procedure. Therefore, the 90 -day mortality rate was $2.8 \%$.

\section{Prognosis and Recurrence}

The median follow-up period was 2,246 days. The majority of the sites of tumor recurrence were hematogenous metastases. In total, 9, 2 and 2 cases had hematogenous, locoregional and both types of recurrences, respectively. Eighteen patients were alive and free of cancer at the last follow-up, while 11 and 2 patients had died of cancer or another cause (acute myocardial infarction and pneumonia in 1 case each), and 5 patients were alive with cancer.

The global 5-year overall survival (OS) rate was $77.8 \%$ in the present series (fig. 1a); the 5 -year OS rates in the patients with complete resection $(\mathrm{n}=33)$ and in those with stage III cancer were $83.3 \%$ (fig. 1 b) and $79.9 \%$ (fig. 1c), respectively; the 5-year OS rate in both the non-SQ and SQ patients was $77.8 \%$ (fig. 2a); the 5-year OS rates in the patients with Ef1-2 and Ef3 response were 72.7 and $81.9 \%$, respectively ( $p=0.55$ ) (fig. $2 \mathrm{~b}$ ), and the 5-year OS rates in the patients with and without downstaging were 83.9 and $53.6 \%$, respectively $(\mathrm{p}=$ 0.39) (fig. 2c).

\section{Discussion}

This study included one expected and one novel finding. First, the non-SQ histology, Ef3 response and downstaging had a tendency to be associated with a favorable prognosis. These phenomena were expected. For example, adenocarcinoma histology has previously been reported to be associated with a more favorable prognosis [15]. Thus, the chemoradiotherapy response would be expected to differ between SQs and adenocarcinomas. Additionally, the data regarding the pathological response were consistent with previous results [16, 17]. The results of several clinical trials have indicated that a pathological response was the most important predictor of long-term survival $[7,18,19]$. Moreover, the favorable trend for cases with downstaging in this study is also consistent with the findings of previous studies $[7,20,21]$.

Second, the treatment-related death in only 1 case and the overall favorable prognosis were somewhat unexpected. Our overall morbidity and mortality rates were exceptional. Previous studies have reported that the rate of postoperative morbidity ranged from 40 to $60 \%$, and the mortality rates ranged from 4 to $20 \%[13,22,23]$. The reported incidences of postoperative morbidity and mortality after induction therapy have varied in different studies, probably due to the sample size, eligibility criteria, the regimens used for preoperative treatment and the experience of the medical staff $[24,25]$. There was a low frequency of morbidity and no 30-day postoperative mortality in our series. These excellent results might be due to selection bias given the retrospective nature of our analysis. Our eligibility requirements included patients at a relatively young age and with a good performance status and adequate organ function. The prolonged survival seen in this study might also partly be explained by these factors.

However, we think that these findings might have been due to a long period between the completion of radiation and surgery. This time lapse was about double that in other reports [26-28]. This might have reduced the preoperative risk and been useful for patient selection. 
In fact, we performed restaging after induction therapy to allow for the selection of the patients who had previously been considered to be unlikely to benefit from surgery [29].

Although complete resection is known to play a central role as a curative treatment for LA-NSCLC after induction chemotherapy [30,31], our survival data were still much better than those in past reports. Careful patient selection is important for multimodality approaches, and the identification of patient subgroups who are likely to have an advantageous outcome after preoperative chemoradiation, and a narrowing of inclusion criteria to further increase the proportion of patients who go on to have surgery with complete resection should be the aim [21]. Of note, a complete pathological response was found in about half of our cases. Our pathological response rate was relatively high. Sakai et al. [9] reported that the overall response rate was $91 \%$, based on an analysis of 32 patients with stage III unresectable NSCLC. In fact, 27 among the 36 cases in our series were treated with DTX and CBDCA. The use of a radiation sensitizer, which both of these drugs are, might have led to the higher pathological response rate [32, 33]. For this reason, the decisions regarding treatment should be made in a multidisciplinary setting that includes a thoracic surgeon, a medical oncologist and a radiation oncologist to determine the most appropriate treatment strategy [34].

There are several limitations that must be taken into account when considering the present findings. These include the retrospective nature of the study and the fact that it was carried out at a single institution. There were imbalances in the patients' characteristics that cannot be excluded due to the small number of patients and the selection bias for the patients. Furthermore, this study included T3N0 cases (so-called Pancoast tumors). Nevertheless, the current results highlight an important issue, because the long-term outcome of induction chemoradiotherapy followed by surgery for LA-NSCLC was favorable. A complete pathological response was found in about half of our cases. This strategy is feasible, and was associated with low morbidity and high resectability rates, suggesting that it contributed to improving the treatment results.

\section{Disclosure Statement}

There are no conflicts of interest or financial interests for any of the authors in association with this study.

\section{References}

1 Kato M, Onishi H, Matsumoto K, et al: Preoperative chemoradiotherapy using cisplatin plus S-1 can induce downstaging in patients with locally advanced (stage III) non-small-cell lung cancer. Anticancer Res 2012;32:5099-5104.

-2 Uramoto H, Shimokawa H, Hanagiri T, et al: Factors predicting the surgical outcome in patients with T3/4 lung cancer. Surg Today, in press.

-3 Suzuki M, Yoshida S, Moriya Y, et al: Surgical outcomes of newly categorized peripheral T3 non-small cell lung cancers: comparisons between chest wall invasion and large tumors $(>7 \mathrm{~cm})$. Interact Cardiovasc Thorac Surg 2010;11:420-424.

4 Endoh H, Yamamoto R, Satoh Y, et al: Risk analysis of pulmonary resection for elderly patients with lung cancer. Surg Today 2013;43:514-520.

-5 Yoneda S, Yamamoto M, Sakura M, et al: Induction chemoradiotherapy followed by surgery for stage III nonsmall cell lung cancer. Jpn J Clin Oncol 1993;23:173-177.

6 Yamamoto M, Mikami I, Nishimura H, et al: Evaluation of neo-adjuvant therapy (NAT) applied to clinical stage III of lung cancer at our center. Nippon Kyoubu Geka Gakkai Zasshi 1996;44:317-319.

-7 Albain KS, Swann RS, Rusch VW, et al: Radiotherapy plus chemotherapy with or without surgical resection for stage III non-small-cell lung cancer: a phase III randomised controlled trial. Lancet 2009;374:379-386. 
Uramoto et al.: The Long-Term Outcomes of Induction Chemoradiotherapy Followed by Surgery for Locally Advanced Non-Small Cell Lung Cancer

8 Uramoto H, Nakanishi R, Fujino Y, et al: Prediction of pulmonary complications after a lobectomy in patients with non-small cell lung cancer. Thorax 2001;56:59-61.

-9 Sakai H, Yoneda S, Kobayashi K, et al: Phase II study of bi-weekly docetaxel and carboplatin with concurrent thoracic radiation therapy followed by consolidation chemotherapy with docetaxel plus carboplatin for stage III unresectable non-small cell lung cancer. Lung Cancer 2004;43:195-201.

10 Phernambucq EC, Spoelstra FO, Verbakel WF, et al: Outcomes of concurrent chemoradiotherapy in patients with stage III non-small-cell lung cancer and significant comorbidity. Ann Oncol 2011;22:132-138.

-11 Saitoh J, Saito Y, Kazumoto T, et al: Concurrent chemoradiotherapy followed by consolidation chemotherapy with bi-weekly docetaxel and carboplatin for stage III unresectable, non-small-cell lung cancer: clinical application of a protocol used in a previous phase II study. Int J Radiat Oncol Biol Phys 2012;82:1791-1796.

12 Therasse P, Arbuck SG, Eisenhauer EA, et al: New guidelines to evaluate the response to treatment in solid tumors. European Organization for Research and Treatment of Cancer, National Cancer Institute of the United States, National Cancer Institute of Canada. J Natl Cancer Inst 2000;92:205-216.

13 Fujita S, Katakami N, Takahashi Y, et al: Postoperative complications after induction chemoradiotherapy in patients with non-small-cell lung cancer. Eur J Cardiothorac Surg 2006;29:896-901.

14 Japan Lung Cancer Society: General Rule for Clinical and Pathological Record of Lung Cancer, ed 6. Tokyo, Kanehara, 2010, pp 176-177.

15 Asamura H, Goya T, Koshiishi Y, et al: A Japanese Lung Cancer Registry study: prognosis of 13,010 resected lung cancers. J Thorac Oncol 2008;3:46-52.

-16 Mouillet G, Monnet E, Milleron B, et al; Intergroupe Francophone de Cancérologie Thoracique (IFCT): Pathologic complete response to preoperative chemotherapy predicts cure in early-stage non-small-cell lung cancer: combined analysis of two IFCT randomized trials. J Thorac Oncol 2012;7:841-849.

17 Pataer A, Kalhor N, Correa AM, et al: Histopathologic response criteria predict survival of patients with resected lung cancer after neoadjuvant chemotherapy. J Thorac Oncol 2012;7:825-832.

18 Choi NC, Carey RW, Daly W, et al: Potential impact on survival of improved tumor downstaging and resection rate by preoperative twice-daily radiation and concurrent chemotherapy in stage IIIA non-smallcell lung cancer. J Clin Oncol 1997;15:712-722.

19 Eberhardt WE, Albain KS, Pass H, et al: Induction treatment before surgery for non-small cell lung cancer. Lung Cancer 2003;42:S9-S14.

20 Rosell R, Lopez-Cabrerizo MP, Astudillo J: Preoperative chemotherapy for stage IIIA non-small cell lung cancer. Curr Opin Oncol 1997;9:149-155.

21 Thomas M, Rübe C, Hoffknecht P, et al: Effect of preoperative chemoradiation in addition to preoperative chemotherapy: a randomised trial in stage III non-small-cell lung cancer. Lancet Oncol 2008;9:636-648.

22 Van Schil P, Meerbeeck JV, Kramer G, et al: Morbidity and mortality in the surgery arm of EORTC 08941 trial. Eur Respir J 2005;26:192-197.

23 Stamatis G, Djuric D, Eberhardt W, et al: Postoperative morbidity and mortality after induction chemoradiotherapy for locally advanced lung cancer: an analysis of 350 operated patients. Eur J Cardiothorac Surg 2002;22:292-297.

24 Martin J, Ginsberg RJ, Abolhoda A, et al: Morbidity and mortality after neoadjuvant therapy for lung cancer: the risks of right pneumonectomy. Ann Thorac Surg 2001;72:1149-1154.

25 Sonett JR, Krasna MJ, Suntharalingam M, et al: Safe pulmonary resection after chemotherapy and high-dose thoracic radiation. Ann Thorac Surg 1999;68:316-320.

26 Toyooka S, Kiura K, Shien K, et al: Induction chemoradiotherapy is superior to induction chemotherapy for the survival of non-small-cell lung cancer patients with pathological mediastinal lymph node metastasis. Interact Cardiovasc Thorac Surg 2012;15:954-960.

27 Taylor NA, Liao ZX, Cox JD, et al: Equivalent outcome of patients with clinical Stage IIIA non-small-cell lung cancer treated with concurrent chemoradiation compared with induction chemotherapy followed by surgical resection. Int J Radiat Oncol Biol Phys 2004;58:204-212.

28 Katakami N, Tada H, Mitsudomi T, et al: A phase 3 study of induction treatment with concurrent chemoradiotherapy versus chemotherapy before surgery in patients with pathologically confirmed N2 stage IIIA nonsmall cell lung cancer (WJTOG9903). Cancer 2012;118:6126-6135.

-29 Kueng MM, Betticher DC: Treatment in resectable, locally advanced NSCLC: which is the best approach? Expert Rev Respir Med 2008;2:655-661.

-30 Song WA, Zhou NK, Wang W, et al: Survival benefit of neoadjuvant chemotherapy in non-small cell lung cancer: an updated meta-analysis of 13 randomized control trials. J Thorac Oncol 2010;5:510-516.

-31 Garrido P, González-Larriba JL, Insa A, et al: Long-term survival associated with complete resection after induction chemotherapy in stage IIIA (N2) and IIIB (T4N0-1) non small-cell lung cancer patients: the Spanish Lung Cancer Group Trial 9901. J Clin Oncol 2007;25:4736-4742.

32 Manson KA, Hunter NR, Milas M, et al: Docetaxel enhances tumor radioresponse in vivo. Clin Cancer Res 1997;3:2431-2438.

-33 Douple EB, Richmond RC, O'Hara JA, et al: Carboplatin as a potentiator of radiation therapy. Cancer Treat Rev 1985;12(suppl A):111-124.

34 Koshy M, Fedewa SA, Malik R, et al: Improved survival associated with neoadjuvant chemoradiation in patients with clinical stage IIIA(N2) non-small-cell lung cancer. J Thorac Oncol 2013;8:915-922. 
Uramoto et al.: The Long-Term Outcomes of Induction Chemoradiotherapy Followed

Table 1. Patient characteristics

\begin{tabular}{|c|c|}
\hline \multicolumn{2}{|l|}{ Gender } \\
\hline Male & 32 \\
\hline Female & 4 \\
\hline Mean age (range), years & $57.6(29-74)$ \\
\hline \multicolumn{2}{|l|}{ Histology } \\
\hline $\mathrm{AD}$ & 19 \\
\hline SQ & 15 \\
\hline ADSQ & 1 \\
\hline LCNEC & 1 \\
\hline \multicolumn{2}{|l|}{ Clinical stage } \\
\hline IIB & 8 \\
\hline IIIA & 16 \\
\hline IIIB & 12 \\
\hline \multicolumn{2}{|l|}{ Regimen of chemotherapy } \\
\hline CBDCA/DTX & 27 \\
\hline CDDP/DTX & 8 \\
\hline CDDP/VP16 & 1 \\
\hline \multicolumn{2}{|l|}{ Pulmonary resection } \\
\hline Lobectomy & 31 \\
\hline Bilobectomy & 2 \\
\hline Pneumonectomy & 2 \\
\hline Partial resection & 1 \\
\hline \multicolumn{2}{|l|}{ Curability } \\
\hline Complete resection & 34 \\
\hline Incomplete resection & 2 \\
\hline \multicolumn{2}{|l|}{ Response } \\
\hline CR & 1 \\
\hline PR & 32 \\
\hline SD & 3 \\
\hline \multicolumn{2}{|c|}{ Pathological responsiveness } \\
\hline Ef1 & 7 \\
\hline Ef2 & 12 \\
\hline Ef3 & 17 \\
\hline
\end{tabular}

Values represent numbers of patients unless stated otherwise. $\mathrm{AD}=$ Adenocarcinoma; $\mathrm{ADSQ}=$ adenosquamous carcinoma; $\mathrm{LCNEC}=$ largecell neuroendocrine carcinoma; $\mathrm{CR}=$ complete response; $\mathrm{PR}=$ partial response; $\mathrm{SD}=$ stable disease. 
Uramoto et al.: The Long-Term Outcomes of Induction Chemoradiotherapy Followed

Table 2. Clinical and pathological responses

\begin{tabular}{ccccc}
\hline \multicolumn{5}{c}{ Pathological responses } \\
\cline { 2 - 5 } & $\begin{array}{l}\text { Ef1 } \\
(\mathrm{n}=7)\end{array}$ & $\begin{array}{l}\text { Ef2 } \\
(\mathrm{n}=12)\end{array}$ & $\begin{array}{l}\text { Ef3 } \\
(\mathrm{n}=17)\end{array}$ & $\begin{array}{c}\text { total } \\
(\mathrm{n}=36)\end{array}$ \\
\hline \multicolumn{7}{c}{ Clinical responses } \\
CR & 0 & 0 & 1 & 1 \\
PR & 6 & 10 & 16 & 32 \\
SD & 1 & 2 & 0 & 3 \\
\hline \multicolumn{2}{l}{ CR = Complete response; PR = partial response; SD = stable disease. } \\
\hline
\end{tabular}

Table 3. Relationship between the clinical diagnosis and pathological downstaging

\begin{tabular}{llll}
\hline Clinical stage & Pathological stage & $\mathrm{n}$ & Downstaging, \% \\
\hline IIB $(\mathrm{n}=8)$ & 0 & 3 & 50 \\
& I & 1 & \\
& IIB & 3 & \\
IIIA $(\mathrm{n}=16)$ & IIIA & 1 & \\
& 0 & 9 & 88 \\
IIIB $(\mathrm{n}=12)$ & I & 5 & \\
& IIIA & 2 & \\
& I & 5 & \\
& IIIB & 3 & 67 \\
\hline
\end{tabular}

Table 4. Outcome after surgery

\begin{tabular}{ll}
\hline Morbidity & $4(11.1 \%)$ \\
Atelectasis & 1 \\
Chylothorax & 1 \\
Prolonged air leak & 1 \\
Empyema & 1 \\
90 -day mortality & $1(2.8 \%)$ \\
Hemoptysis & 1 \\
\hline
\end{tabular}


Uramoto et al.: The Long-Term Outcomes of Induction Chemoradiotherapy Followed by Surgery for Locally Advanced Non-Small Cell Lung Cancer
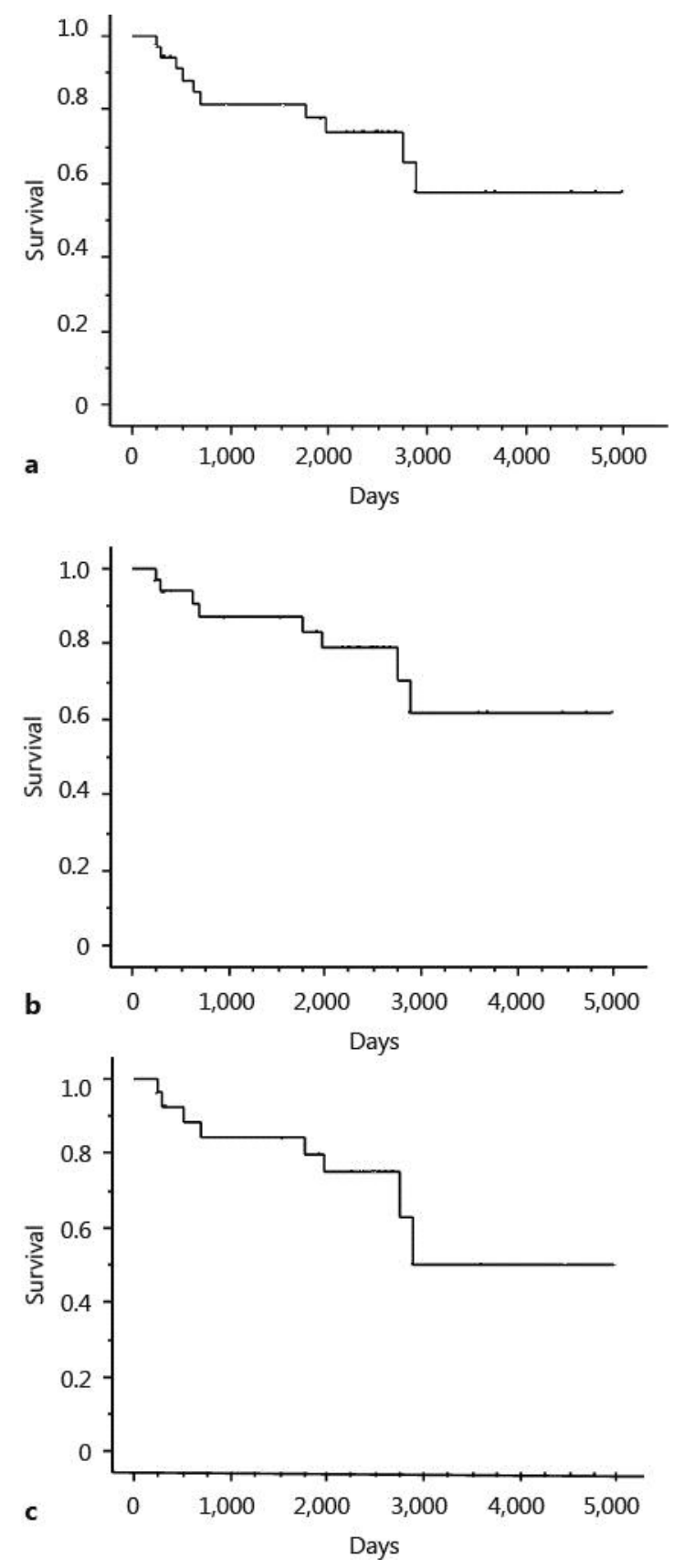

Fig. 1. a The results of a Kaplan-Meier analysis of the LA-NSCLC patients who received induction chemoradiotherapy followed by surgery. The 5-year OS rate in the patients was $77.8 \%$. b The 5-year OS rate in the patients with complete resection was $83.3 \%$. c The 5-year OS rate in the patients with stage III disease was $79.9 \%$. 
Uramoto et al.: The Long-Term Outcomes of Induction Chemoradiotherapy Followed by Surgery for Locally Advanced Non-Small Cell Lung Cancer
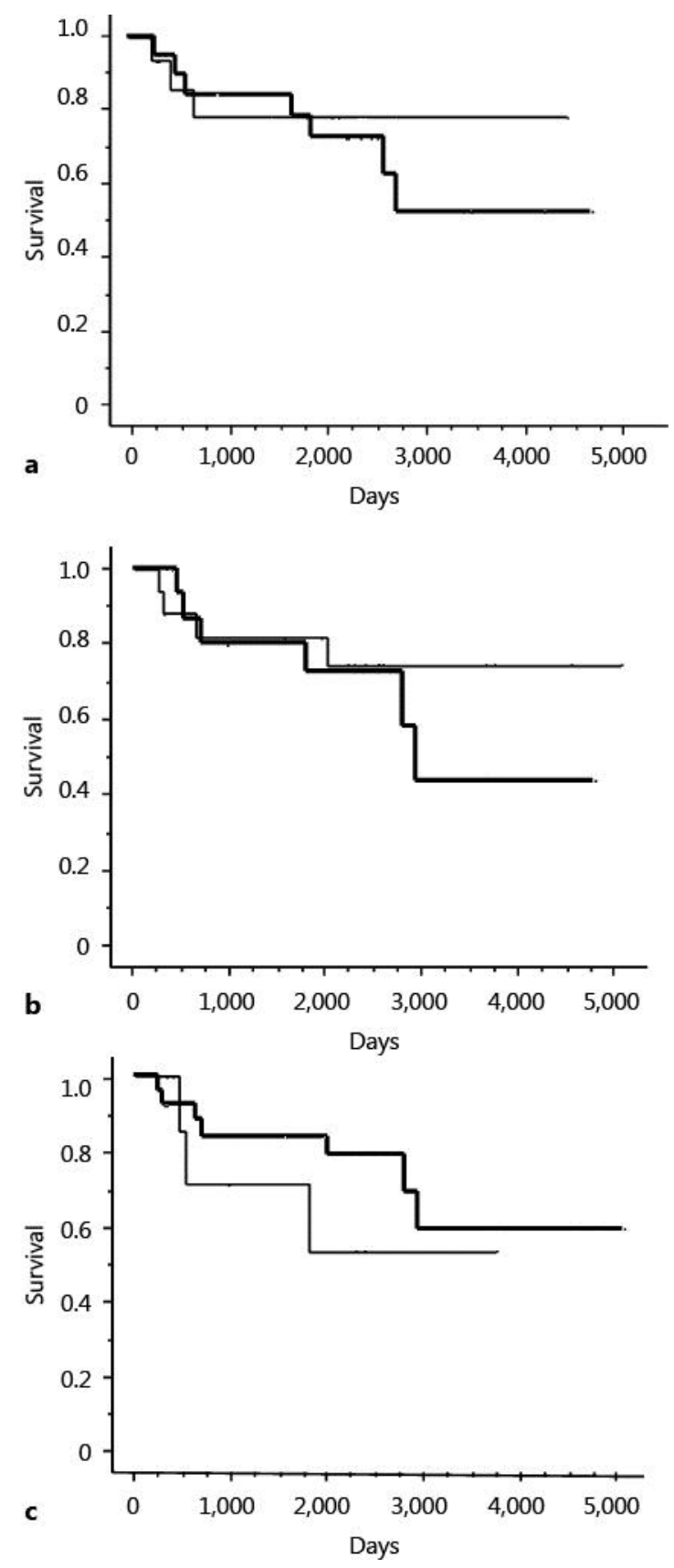

Fig. 2. The results of Kaplan-Meier analyses of the OS of patients stratified by histology, pathological response and downstaging. a The 5-year OS rate in both the non-SQ (thin line) and SQ (thick line) patients was 77.8\%. b The 5-year OS rates in the Ef1-2 (thick line) and Ef3 (thin line) patients were 72.7 and $81.9 \%$, respectively $(\mathrm{p}=0.55)$. c The 5-year OS rates in the patients with downstaging (thick line) and without downstaging (thin line) were 83.9 and $53.6 \%$, respectively $(p=0.39$ ). 